Please do not remove this page

RMIT

UNIVERSITY

\title{
Perceptually lossless image coding of digital medical mammograms with arithmetic visual pruning
}

Wu, David; Tan, Damian; Wu, Henry

https://researchrepository.rmit.edu.au/esploro/outputs/9921862547101341/filesAndLinks?institution=61RMIT_INST\&index=null

Wu, D., Tan, D., \& Wu, H. (2006). Perceptually lossless image coding of digital medical mammograms with arithmetic visual pruning. Proceedings of 2006 International Symposium on Intelligent Signal Processing and Communication Systems, 935-938. https://doi.org/10.1109/ISPACS.2006.364794

Published Version: https://doi.org/10.1109/ISPACS.2006.364794

Repository homepage: https://researchrepository.rmit.edu.au

(C) IEEE 2006

Downloaded On 2023/04/26 14:56:14 +1000

Please do not remove this page 


\title{
PERCEPTUALLY LOSSLESS IMAGE CODING OF DIGITAL MEDICAL MAMMOGRAMS WITH ARITHMETIC VISUAL PRUNING
}

\author{
David Wu, Damian M. Tan and Hong Ren Wu \\ School of Electrical \& Computer Engineering \\ Royal Melbourne Institute of Technology \\ Melbourne, Victoria, Australia, 3001 \\ Email: Henry.Wu@rmit.edu.au
}

\begin{abstract}
A method of encoding digital medical mammograms is presented in this paper. Based on the JPEG2000 coding framework and embedded with an advanced perceptual distortion measure, the proposed coder identifies and removes visually insignificant/irrelevant information, so as to achieve perceptually lossless quality. Compared with the state-of-the-art JPEG compliant LOCO lossless coder and JPEG2000 lossless coder, current results have shown compression ratio gains in bit-rate without any loss of visual fidelity.
\end{abstract}

\section{INTRODUCTION}

Early detection of cancer in breasts, performed through mammography, remains to be a critical solution in reducing the mortality rate [1], [2]. Coupled with the advances of digital technology, various applications such as Telemammography [1] have risen and offer the benefits of faster and accurate diagnosis, thus reaching out to those in need in geographically remote locations. Aside from high bit-depth and resolution, mammograms require digitization spot sizes of at least $50 \mu \mathrm{m}$ for detailed and accurate diagnoses[3]. Nonetheless, digitisation enables clinically critical mammographic information to be indefinitely stored without harm from the harsh external environment and can be transmitted to any geographical location with relative ease. A problem associated with this, however, is the large storage space and high bandwidth requirements [4]. An approach to alleviating this problem is through image compression, which has been an area of much controversy. Generally, the compression of digital images can be categorised into two types, the first being reversible (lossless) image coding and the second being irreversible (lossy) image coding [5]. Reversible image coding encodes an image without any loss of information throughout its entire process. They, however, are unfortunately limited in their compression ratio gain, having ratios at most between $3: 1$ up to $4: 1[6]$. Hence the need for irreversible image coding schemes, exchanging image information integrity for compression ratio gain, which inevitably can lead to visible distortions if the process is not controlled or monitored. Perceptually lossless image coding (PLIC) provides alternative method for encoding medical images. The aim of PLIC is to encode a medical image to a degree such that it is indistinguishable from the original[7], offering the best of both worlds; having greater compression ratio gain without any loss in visual fidelity. Previously, a work on PLIC[8] was presented for MRI[9] and CT[10] images. This paper presents a novel coder for encoding digital mammograms. Mammograms differ from from MRI and CT, in that the soft tissue of breasts are captured and they require a greater level of precision and accuracy for critical diagnosis[3]. This work encompasses an identical vision model as that in [11], to identify visually insignificant/irrelevant information, and is embedded into an advanced arithmetic visual pruning (AVP) algorithm to effectively remove visually insignificant/irrelevant information. In addition, the pruning algorithm can be integrated into any Wavelet based coding scheme without disrupting bit-stream compliance and thus would not require any specialised decoder. In this paper, AVP is built into the core JPEG2000[12] coding engine. This is particularly favourable, since JPEG 2000 is accepted as a medical image coder in the Digital Communications in Medicine (DICOM) standard [13].

This paper is presented as follows. Section II will describe the proposed coder, covering the coder design and its pruning algorithm. Section III then presents and discusses the results and, finally, Section IV draws to light the discussions given in this paper.

\section{PROPOSED CODER}

\section{II-A. Arithmetic Visual Pruning}

Let us consider the problem of encoding a transformed image, $\mathrm{X}$ to a point such that the difference from its processed image, $\hat{\mathbf{X}}$, is less than or equal to the JustNot-Noticeable-Difference threshold, a point whereby differences between the two images are visually indistin- 
guishable, i.e., Perceptually Lossless. We can define this problem as follows,

$$
d_{v}(\mathbf{X}, \hat{\mathbf{X}}) \leq J N N D
$$

where $d_{v}$ is a perceptual quality/distortion measure and $J N N D$ is the Just-Not-Noticeable-Difference threshold. The unknown here is what should $\hat{\mathrm{X}}$ be or how much should $\mathbf{X}$ be quantised. An alternative representation is $\hat{\mathbf{X}}[m, n]=p_{m, n} \times \mathbf{X}[m, n]$, with $p_{m, n}$ being a percentage between 0.0 and 1.0, inclusive, for each pixel at a location $(m, n)$. This is then a problem of how to find an optimal

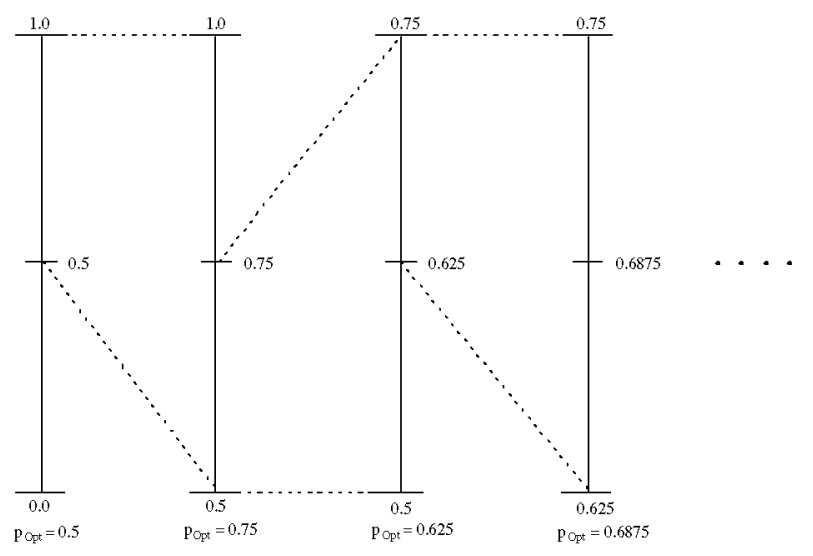

Fig. 1. The process of determining $p_{O p t}$.

value $p_{O p t_{m, n}}$, such that Equation 1 remains true. One approach is through varying step sizes of $p_{m, n}$, which can be computationally intensive. An alternative is given through a modified form of the binary search algorithm (Figure 1).

$$
\begin{gathered}
\quad S\left(T_{D}, D_{T}, R_{M}, R_{L}, R_{H}\right)= \\
\begin{cases}0 & , \frac{R_{L}+R_{H}}{2} \leq B_{\text {zero }} \\
S\left(T_{D}, D_{T}, \frac{R_{L}+R_{M}}{2}, R_{L}, R_{M}\right) & , D_{T}<T_{D} \\
S\left(T_{D}, D_{T}, \frac{R_{M}+R_{H}}{2}, R_{M}, R_{H}\right) & , D_{T}>T_{D} \\
R_{M} & ,\left|D_{T}-T_{D}\right| \leq B_{\text {equal }} \\
1 & , \frac{R_{L}+R_{H}}{2} \geq B_{\text {one }}\end{cases}
\end{gathered}
$$

where $T_{D}, D_{T}, R_{M}, R_{L}$ and $R_{H}$ are respectively, a subjectively measured JNND threshold, the distortion measure determined by $d_{v}$, a midpoint representing $p_{m, n}$, a lower bound of a given range of values and an upper bound of a given range of values. Initially, $R_{M}$ is set to 0.5 , $R_{L}$ and $R_{H}$ are set to 0.0 and 1.0 , respectively. $B_{\text {zero }}$, $B_{\text {one }}$ and $B_{\text {equal }}$ are arbitrary set precision bounds. In this paper, $B_{\text {zero }}=0.000001, B_{\text {one }}=1-B_{\text {zero }}$ and $B_{\text {equal }}=B_{\text {zero }}$. These precision bounds are necessary, providing a stopping point in the algorithm, due to the limitation in finite precision. Therefore it is necessary to initially subtract $B_{\text {zero }}$ from $T_{D}$, so that Equation 1 remains true.

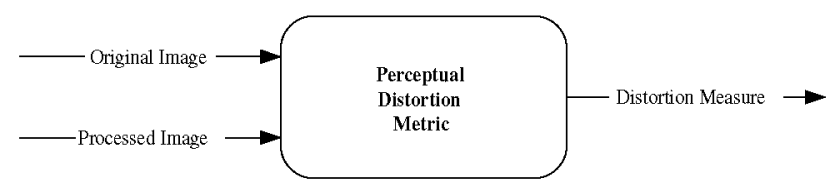

Fig. 2. A generalised concept of a perceptual distortion metric. A detailed treatment and description of these measures are discussed in [14].

\section{II-B. Perceptual Distortion Metric}

Traditional metrics such as Mean Squared Error (MSE), Peak-Signal-Noise Ratio (PSNR) have served as one of the basic means for quantifying distortion/quality[15]. However, while they may be computationally simple, they do not correlate well with human perception[16]. Hence, the need for contemporary metrics that incorporate the characteristics of the Human Visual System(HVS). The effectiveness of this approach has been demonstrated in several pioneering works in picture quality quality/impairment assessment [14], [17], [18]. In this paper, the proposed coder(PC), employs an advanced human vision model described in [11], [19]. The model is a multi-channel function, based on the Contrast Gain Control[17] within the Wavelet domain; taking into account of visual masking within a sub-band (intra frequency masking) and between orientations (inter orientation masking) [19]. The effectiveness of this model was demonstrated for medical image compression in [11]. For a detailed description of the model, the reader is referred to [11], [19].

\section{II-C. Determining JNND}

JNND is defined as a threshold where no differences can be perceived between a processed and a reference image. To effectively determine the JNND, the process is performed in two stages in the Wavelet domain. The first stage approximates the threshold and the second stage fine tunes the threshold. In the first stage, an image is uniformly degraded in the Wavelet domain for each orientation, $\theta$ and sub-band level, $l$, with a given percentage $p_{l, \theta}$, between 0.0 and 1.0. The processed image is then reverted back into the image domain and is segmented into nonoverlapping blocks of size $32 \times 32$ pixels. By comparing with the original image, $p_{l, \theta}$ is adjusted such that visible distortions are not visible in the processed image. The segmented block approach is necessary, so as to effectively capture localised variations in image quality. The most visually sensitive block, to distortion, is then subjected to the distortion measure, $d_{v}$, in equation 1 , which provides the subjectively measured JNND threshold, $T_{D}$. Hence, $T_{D}=T_{D}[l, \theta]$, is a set of thresholds for each level and orientation. The second stage fine tunes the thresholds $T_{D}$, by employing the temporal sensitivities of the HVS. Here, $T_{D}$ is adjusted accordingly and the processed image is 
overlaid on the original image and flipped back and forth, so as to ensure 'distortion flickers' are not visible.

\section{II-D. JPEG 2000 Adaptation}

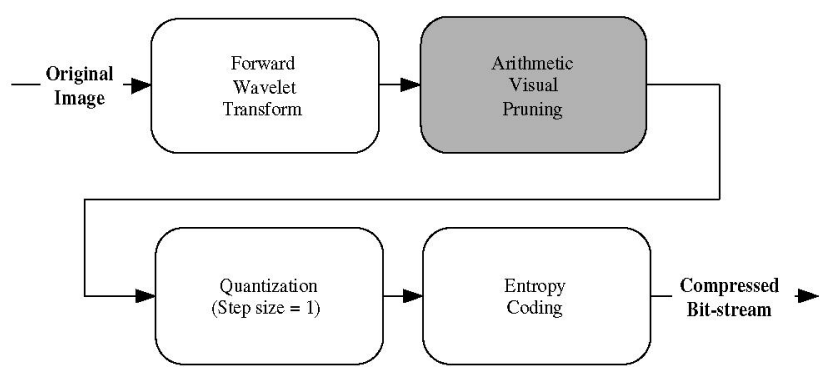

Fig. 3. Integration of the AVP into a generalised JPEG 2000 coding structure[12]. Here, the step size of the scalar quantizer is set to 1 .

In order to maintain bit-stream compliance, the AVP algorithm is immediately integrated after the forward Wavelet transform in the JPEG 2000[12] coding architecture (Figure 3). In addition, so as to ensure that only visually insignificant/irrelevant information is removed, the base step size of the scalar quantizer is set to 1 .

\section{PERformance evaluation}

To evaluate the performance of the $\mathrm{PC}$, it is compared against the state-of-the-art JPEG-LS compliant LOCO lossless coder (LOCO)[20] and JPEG 2000 lossless mode[12] (J2KL), of which each compressed 24 breast images. These breast images were obtained from the University of South Florida Mammogram Database[21]. In terms of coding performance, both LOCO and J2KL performed relatively the same. On the other hand, the PC on average performed better with approximately $37 \%$ compression ratio gain over LOCO and J2KL. In comparison with the uncompressed images, there was an average compression ratio gain of $77 \%$ and $62 \%$ for $16 \mathrm{bpp}$ and 12 bpp images, respectively (Table I). In terms of computational complexity, the LOCO coder achieves the lowest complexity and the PC had a much higher complexity, due to the addition of the visual pruning function. Nevertheless and more importantly, there was no loss in visual fidelity in the images compressed by the PC (Figure 4).

\section{CONCLUSION}

This paper presented a novel method of encoding digital mammograms at a perceptually lossless quality. Embedded with an advanced human vision model and built on the JPEG2000[12] framework, the PC can identify and remove visually irrelevant/insignificant information. In addition, it does not require any specialised decoder. The advantages are two folds, the first is that it does not disrupt the

\begin{tabular}{|c|c|c|c|c|}
\hline Image & Dimensions & \multicolumn{3}{|c|}{ Bitrate (bpp) } \\
\cline { 3 - 5 } Name & (pixels) & LOCO & J2KL & PC \\
\hline \hline Breast 1 & $1979 \times 4349$ & 5.980 & 5.928 & 3.261 \\
\hline Breast 2 & $2000 \times 5024$ & 6.228 & 6.204 & 3.569 \\
\hline Breast 3 & $1874 \times 4334$ & 5.618 & 5.557 & 2.887 \\
\hline Breast 4 & $2129 \times 5084$ & 6.266 & 6.238 & 3.622 \\
\hline Breast 5 & $2549 \times 5339$ & 6.284 & 6.241 & 3.606 \\
\hline Breast 6 & $2759 \times 5624$ & 6.879 & 6.839 & 4.264 \\
\hline Breast 7 & $2444 \times 5384$ & 6.496 & 6.485 & 3.921 \\
\hline Breast 8 & $2594 \times 5609$ & 6.976 & 6.950 & 4.383 \\
\hline Breast 9 & $2928 \times 4608$ & 6.895 & 7.039 & 4.602 \\
\hline Breast 10 & $2928 \times 4608$ & 6.867 & 7.003 & 4.560 \\
\hline Breast 11 & $2896 \times 4592$ & 6.926 & 7.046 & 4.585 \\
\hline Breast 12 & $2920 \times 4584$ & 6.890 & 7.025 & 4.567 \\
\hline Breast 13 & $2888 \times 4616$ & 6.991 & 7.121 & 4.664 \\
\hline Breast 14 & $2816 \times 4664$ & 6.989 & 7.132 & 4.677 \\
\hline Breast 15 & $2600 \times 4656$ & 6.972 & 7.090 & 4.627 \\
\hline Breast 16 & $2536 \times 4680$ & 6.998 & 7.144 & 4.690 \\
\hline Breast 17 & $2672 \times 4648$ & 6.902 & 6.998 & 4.527 \\
\hline Breast 18 & $2664 \times 4680$ & 6.938 & 7.057 & 4.591 \\
\hline Breast 19 & $2816 \times 4704$ & 6.905 & 7.013 & 4.540 \\
\hline Breast 20 & $2864 \times 4648$ & 6.903 & 7.012 & 4.540 \\
\hline Breast 21 & $2616 \times 4672$ & 6.902 & 7.013 & 4.543 \\
\hline Breast 22 & $2424 \times 4664$ & 6.876 & 6.973 & 4.498 \\
\hline Breast 23 & $2680 \times 4656$ & 6.868 & 6.957 & 4.478 \\
\hline Breast 24 & $2752 \times 4680$ & 6.852 & 6.951 & 4.471 \\
\hline \multicolumn{2}{|c|}{ AVERAGE } & 6.725 & 6.792 & 4.278 \\
\hline
\end{tabular}

Table I. Shows the coding performance, in terms of bitrate, of LOCO, J2KL and PC. All Breast images have bit-depths of $12 \mathrm{bpp}$, except Breast 1 to Breast 8 , which have bitdepths of $16 \mathrm{bpp}$.

bit-stream compliance of JPEG2000, hence, subsequently compliance with the DICOM standard is maintained. The second is that it can be integrated into Wavelet based image coder. More importantly, the current results have shown that the PC outperforms its lossless counterparts with any loss of visual fidelity.

\section{REFERENCES}

[1] E. Sheybani and R. Sankar, "ATMTN: a telemammography network architecture," IEEE Transactions on Biomedical Engineering, vol. 49, no. 12, pp. 1438 - 1443, 2002.

[2] S. K. Moore, "Better breast cancer detection," IEEE Spectrum, vol. 38, no. 5, pp. 50-54, 2001.

[3] M. Penedo, W. A. Pearlman, P. G. Tahoces, M. Souto, and J. J. Vidal, "Region-Based Wavelet Coding Methods for Digital Mammography," IEEE Transactions on Medical Imaging, vol. 22, no. 10, pp. 1288-1296, Oct 2003.

[4] X. Zhou, H. Huang, and S. Lou, "Authenticity and integrity of digital mammography images," IEEE 

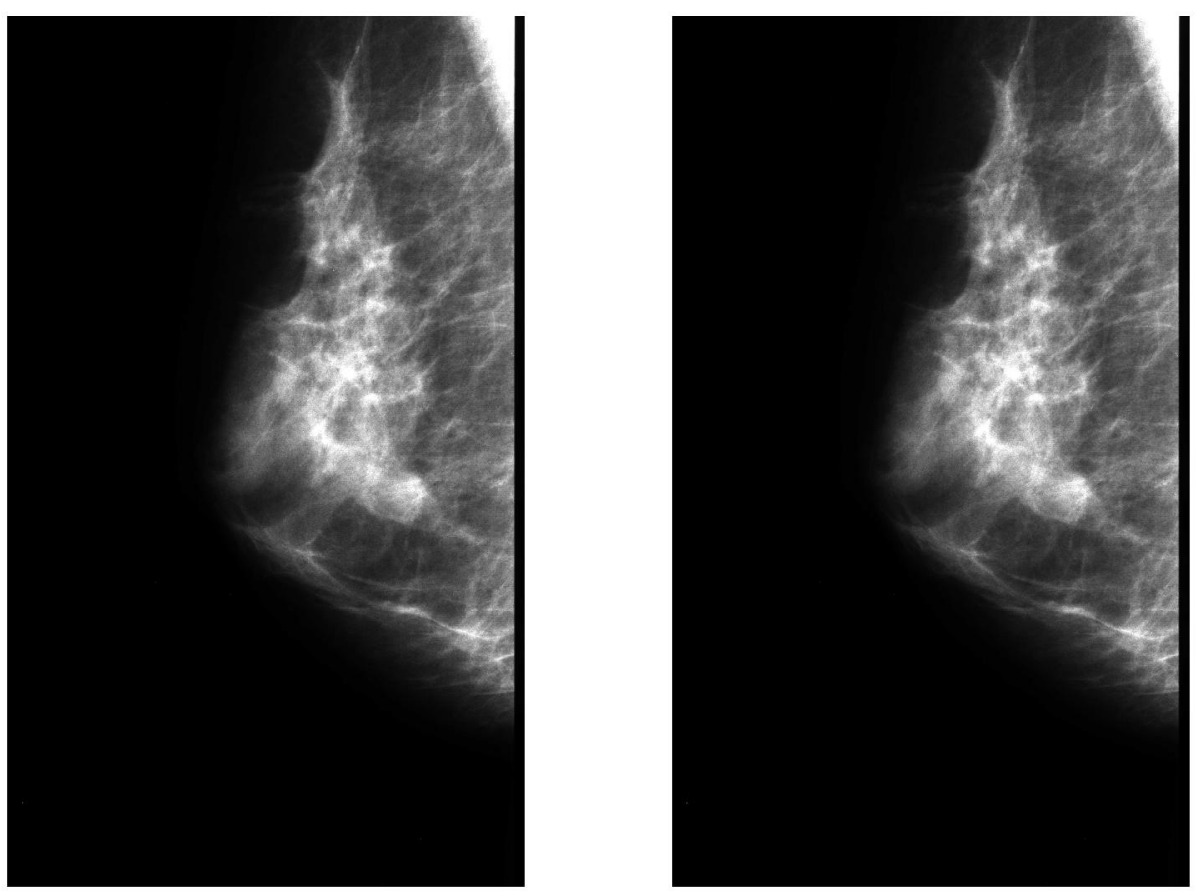

Fig. 4. Image: Breast 2; Left: Original; Right: Proposed

Transactions on Medical Imaging, vol. 20, no. 8, pp. 784 - 791, 2001.

[5] R. C. Gonzalez and R. E. Woods, Digital Image Processing, 2nd ed. Prentice Hall, Inc., 2002.

[6] B. J. Erickson, A. Manduca, P. Palisson, K. R. Persons, F. Earnest, V. Savcenko, and N. J. Hangiandreou, "Wavelet Compression of Medical Images," Radiology, vol. 206, no. 3, pp. 599 - 607, 1998.

[7] A. B. Watson, "Receptive Fields and Visual Represtations," in SPIE Proceedings, vol. 1077. SPIE, 1989 , pp. 190-197.

[8] D. Wu, D. Tan, and H. R. Wu, "A New Visual Pruning Algorithm For Perceptually Lossless Medical Image Coding," Proceedings of the Second International Workshop on Video Processing and Quality Metrics for Consumer Electronics, 2006.

[9] R. Archarya, R. Wasserman, J. Stevens, and C. Hinojosa, "Biomedical Imaging Modalities: A Tutorial," Computerized Medical Imaging and Graphics, vol. 19, no. 1, pp. 3 - 25, 1995.

[10] Kak, A. C. and Slaney, M., Principals of Computerized Tomographic Imaging. IEEE Press, 1987.

[11] D. Wu, D. Tan, M. Baird, J. DeCampo, C. White, and $\mathrm{H}$. R. Wu, "Perceptually Lossless Medical Image Coding," IEEE Transactions of Medical Imaging, vol. 25, no. 3, pp. $335-344,2006$.

[12] D. Taubman and M. W. Marcellin, JPEG2000: Image compression fundamentals, standard and practice, 1st ed. Kluwer Academic Plublishers, 2002.

[13] ACR-NEMA, "DICOM Standard," 2006. [Online]. Available: http://medical.nema.org/
[14] H. R. Wu and K. R. Rao, Eds., Digital Video Image Quality and Perceptual Coding, ser. Signal Processing and Communications. CRC Press, 2005.

[15] N. S. Jayant and P. Noll, Digital Coding Of Waveforms, 1st ed. Prentice-Hall,Inc., 1984.

[16] B. Girod, What's Wrong with Mean-squared Error?, ser. Digital Images and Human Vision, A. B. Watson, Ed. MIT Press, 1993.

[17] A. B. Watson and J. A. Solomon, "A Model of Visual Contrast Gain Control and Pattern Masking," Journal of the Optical Society of America A, pp. 2379 - 2391, 1997.

[18] Z. Yu, H. R. Wu, S. Winkler, and T. Chen, "Vision Model Based Impairment Metric To Evaluate Blocking Artifacts in Digital Video," Proceedings of the IEEE, vol. 90, no. 1, pp. 154-169, Jan 2002.

[19] D. M. Tan and H. R. Wu, Perceptual Image Coding, ser. Digital Video Image Quality and Perceptual Coding, H. R. Wu and K. R. Rao, Eds. CRC Press, 2005.

[20] M. Weinberger, G. Seroussi, and G. Sapiro, "The LOCO-I Lossless Image Compression Algorithm: Principles and Standardization into JPEG-LS," IEEE Transaction on Image Processing, vol. 9, pp. 13091324, August 2000, Hewlett-Packard Laboratories Technical Report No. HPL-98-193R1, November 1998, revised October 1999.

[21] M. Heath, K. Bowyer, and D. Kopans, Current status of the Digital Database for Screening Mammography, ser. Digital Mammography. Kluwer Academic Publishers, 1998. 